\title{
Risiken, Schäden, Leistungen, Schicksale - alles nur Papier?!
}

\author{
A. Thommen
}

Nur wenig müssen behandelnde Ärztinnen und Ärzte beachten, um ihren Patientinnen und Patienten guten Service zu bieten und Versicherungsärztinnen/ -ärzten die Arbeit zu erleichtern. Formulare gekonnt ausfüllen und daran denken: Versicherte haben nicht immer recht, die Versicherung hat nicht immer unrecht - und vice versa. Versicherungsärztinnen/-ärzte sollten vermehrt einbezogen werden, weil sie beide Welten kennen, die ärztliche genauso wie die der Versicherungen, und weil sie zwischen beiden vermitteln können.

Handelt es sich um zwei verschiedene Personen auf den diversen Formularen? Name, Adresse Beruf und Geburtsdatum sind identisch: Herr K., Jahrgang 1968, beantragte auf dem Formular aus dem Jahr 2000 eine Versicherung, um das finanzielle Risiko von Todesfall und Invalidität abzusichern. Auf dem zweiten Formular aus dem Jahr 2002 fordert der nun 34jährige Herr K. Invaliditätsleistungen, denn er sei permanent und vollständig arbeits- und erwerbsunfähig. Die Gesellschaftsärztin schaut die von der Hausärztin ausgefüllten Formulare durch, die umfangreichen Untersuchungen der Spezialistinnen/Spezialisten und die aggressiven Briefe des Anwalts des Versicherten. Laut dem von Herrn K. selbst ausgefüllten ersten Antragsformular waren er und seine Vorfahren bis im Jahr 2000 völlig gesund und hatten blande persönliche Anamnesen. Herrn K.s Hausärztin, die ihn seit 1970 kennt, fasst auf dem Formular «Ärztliche Untersuchung» den Zustand des Herrn K. als «Vollkommen gesunder Mann ohne Risiko für Invalidität und Tod» zusammen.

Den Optimismus der Hausärztin teilt die Gesellschaftsärztin nicht: Das Todesfallrisiko beträgt im «besten» Falle leider 100\% - wir alle sterben irgendwann. Und viele von uns haben sogar eine «Übersterblichkeit», welche die Versicherungsmathematiker/innen aufgrund von riesigen Datenbanken ausrechnen: Millionen von Patientendaten sind darin enthalten, die international über Jahrzehnte hinweg erhoben wurden.

Das Risiko, dass eine Invalidität eintritt, ist

Korrespondenz:

Dr. med. Annette Thommen

CONSULDOC

Kluserstrasse 2

CH-4054 Basel

E-Mail: a.r.thommen@tiscali.ch
Mensch «gesund». Ganz extrem gewisse Onkologinnen/Onkologen, deren pessimistischstes Wort «Teilremission» lautet.

Für Versicherungsärztinnen/-ärzte, die Prognosen über Jahrzehnte hinaus stellen müssen, ist die WHO-Gesundheitsdefinition, diese (utopische) totale somatopsychosoziale Perfektion, wünschbar.

Die Hausärztin von Herrn K. ist sorgfältig: Obwohl sie laut Formularfragen nur die Ereignisse der letzten fünf Jahre in der Anamnese des Herrn K. aufführen müsste, erwähnt sie eine Pollinosis, eine Atopieneigung der Familie K., eine «kurzdauernde, leichte reaktiv-depressive Episode 1986», die «durch Wohnorts- und Arbeitsplatzwechsel sowie familiäre Probleme bedingt war» und welche ein «hausärztliches Gespräch heilte». Ausserdem waren da noch «anamnestisch vor vielen Jahren leichte HWS-Beschwerden, jedoch kein Tag AUF, ohne Therapie völlig ausgeheilt», und 1987 «zwei Tage akute leichte Lumbago, nach Bettruhe und NSAR rezidivfrei vollkommen geheilt».

Herr K. hatte im Jahr 2000 eine Police zu einer Normalprämie erhalten, ohne Vorbehalte oder Ausschlussklauseln. 2002 ist Herr K. gemäss Schreiben von Hausärztin und Anwalt invalide. Wundersamerweise stellt sich die Zeit vor 2000 jetzt plötzlich anders dar: «Seit Jahrzehnten», so ist dem Bericht des universitären Rheumatologen zu entnehmen, litt Herr K. unter «stärksten Rückenbeschwerden und einer schweren familiären Allergie auf eine Reihe von Substanzen». Ein habilitierter Neurologe diagnostiziert «seit Jahren bestehende Cephalea mit migränoidem Charakter». Beide Herren vermuten das «anamnestisch bekannte mittelschwere HWS-Beschleunigungstrauma» als Ursache. Beide finden zwar weder in der körperlichen Untersuchung noch in Labor oder Bildgebung mehr als geringste, altersübliche Verschleisszeichen. Aber genau wie der Psychiater und der Neuropsychologe orten sie «glaubhaftes Leiden», verneinen Aggravation und schildern die Konzentrationsstörung, die Antriebslosigkeit und die sensorischen Phänomene, die Herr K. ihnen angab. Herrn K. wurde gekündigt, er ist in Scheidung von der Ehefrau, welche IV-Bezügerin ist und ihn 
wegen Unterhalt und Alimenten für die vier Kinder (Jahrgänge 1988, 1992, 1996 und 2000) verklagt. Die Hausärztin hat Herrn K. von einem Spezialisten zum anderen geschickt, diverse Therapien durchgeführt, ihm Termine von der Sozialarbeiterin über die Budgetberatung bis hin zum RAV vermittelt. Doch Herrn K. geht es nicht besser, und er ist arbeitsunfähig. Aber - so Hausärztin und Anwalt - Herr K. sei «topmotiviert und arbeitswillig». Er sage immer wieder, wie er den Job vermisse und dass er sofort wieder voll einstiege, wenn es ihm nur ein bisschen besserginge. Aber leider sei er momentan noch zu schlecht dran ... Die Hausärztin schildert mit keinem Wort, was Herr K. noch kann bzw. was genau er nicht mehr kann oder was er in seinem Beruf machen musste, aber sie appelliert an die Gesellschaftsärztin, doch bitte für die baldige Auszahlung der Versicherungssumme zu sorgen, da die Abklärungen der Versicherung Herrn K. zusätzlich psychisch belasteten. Um einiges aggressiver ist der Anwalt von Herrn K. - dieser fordert und droht.

Nachdem die Case-Managerin der Versicherung bei ihrem Besuch Herrn K. auf der Leiter antraf, beim Lackieren des Dachhimmels seines Häuschens, konnte Herr K. mit sanftem Druck wieder in das Erwerbsleben integriert werden.

Nicht überzeugt war die Versicherung auch vom Leiden der Frau S., die wenige Monate nach Versicherungsabschluss wegen Multipler Sklerose die Auszahlung der Versicherungssumme beantragte. Immer wieder bat sie in handschriftlichen Briefen um «das mir zustehende Geld». Doch ihr Fall wurde als nicht dringlich eingestuft, und das Dossier wanderte mehr als zwei Jahre bei der Versicherung von Sachbearbeiter zu Sachbearbeiter. Als eine neueingestellte Sachbearbeiterin energisch die «Altlasten» aufarbeitete und die Versicherte anrief, erzählte diese, dass bereits zwei Jahre vor Versicherungsabschluss eine passagere Sehstörung aufgetreten war. Diese war vom Hausarzt nicht weiter abgeklärt und im Antrag nicht erwähnt worden - retrospektiv muss eine Retrobulbärneuritis vermutet werden. Das Formular, mit dem die Versicherungsleistung beantragt wurde, enthielt ausser dem handschriftlichen Vermerk des Hausarztes «V.a. M.S.» nichts. Bagatellisiert und verschleppt: Frau S. hatte eine foudroyant verlaufende Form der Multiplen Sklerose, war bereits trotz intensiver Therapie hochgradig gelähmt, die bildgebenden Methoden zeigten ausgedehnte Demyelinisierungsbefunde und das Liquorlabor typische Banden. Die Versicherungssumme, die daraufhin sofort ausgezahlt wurde, war eminent wichtig für die Kranke...
Diese zwei Fälle sind typisch für das, was Gesellschaftsärztinnen/-ärzte bei ihrer Arbeit sehen. Arbeitsunwillige Schlaumeier beuten das System aus - oft mit Hilfe von engagierten, gutmütigen bis naiven Hausärztinnen/-ärzten und streitbaren Anwältinnen/Anwälten. Wirklich Kranke bekommen nicht unverzüglich die ihnen zustehenden Leistungen, weil ihre Hausärztinnen/-ärzte die Formulare nicht gut ausfüllen oder Versicherungsmitarbeiter/innen nicht «schalten». Als Gesellschaftsärztin wünsche ich mir von den behandelnden Kollegen:

1. Papierkram ist zugegebenermassen lästig. Trotzdem: Bitte füllen Sie die Versicherungsformulare ausführlich, objektiv und sorgfältig aus - sowohl die ärztliche Untersuchung, die zu den Antragsformularen für den Abschluss einer Versicherung gehört, wie auch die Fragebogen, die man Ihnen zustellt, wenn die Versicherten Leistungen beantragen. Das dient Ihren Patientinnen und Patienten, die so von der Versicherung den besten Service erhalten.

2. Bitte drücken Sie sich klar und professionell aus. Verwenden Sie medizinische Fachausdrücke und detaillierte ICD-10-Codes, doch möglichst wenig Abkürzungen. Formulieren Sie nicht vereinfachend oder in Umgangssprache, denn diese Formulare werden nicht von irgendwelchen schlichten Geistern gelesen, sondern von erfahrenen Versicherungsprofis und Gesellschaftsärztinnen/-ärzten. Schreiben Sie nur von Hand, wenn Sie eine sehr gut leserliche Schrift haben (fragen Sie Ihren Apotheker!), denn Gesellschaftsärztinnen/-ärzte rätseln täglich an ärztlichen Hieroglyphen herum.

3. Schildern Sie den Sachverhalt naturwissenschaftlich sachlich, und plädieren Sie nicht anwaltlich. Interpretieren Sie nicht, sondern liefern Sie Fakten. Legen Sie objektive Untersuchungsbefunde, wie bildgebende Methoden, Laboruntersuchungen und Konsilien von Spezialistinnen/Spezialisten, bei. Klären Sie unklare Laborbefunde vorher ab, verlangen Sie radiologisch eindeutige Diagnosen vom Röntgeninstitut. Hat Ihr Patient, Ihre Patientin ein sehr seltenes Leiden, und Sie haben darüber eine wichtige wissenschaftliche Publikation, dann legen Sie diese bei das erleichtert den Gesellschaftsärztinnen/ -ärzten die Arbeit und freut diese.

4. Es ist nicht Ihr Job, über Tarife, Prämien oder Risikoklassen zu entscheiden. Liefern Sie keine versicherungsmedizinischen Beurteilungen oder Prognosen, sondern objektive Daten, die diese ermöglichen. Die Versiche- 
rungsmedizin ist eine Fachdisziplin, die mit erprobten statistischen Verfahren arbeitet. Weder kennen Sie die diversen Versicherungsprodukte noch den Markt, für den die Versicherung die Vertragsbedingungen kalkuliert. Die Versicherungsmathematik gründet ihre Berechnungen auf grosse Kollektive, nicht auf einen einzelnen Fall. Klinische Prognosezahlen können nur nach versicherungsmedizinischer Modifikation verwendet werden. Gesellschaftsärztinnen/-ärzte müssen aufgrund einer Standortbestimmung, die Sie liefern, die Entwicklung über die Laufzeit der Versicherung abschätzen; sie können nicht zuwarten und die Kranken immer wieder einbestellen, wie Sie das tun können.

5. Vertrauen Sie bei der Beurteilung der Arbeitsfähigkeit nicht nur auf das, was Patientinnen und Patienten Ihnen von ihrem Arbeitsplatz erzählen. Erheben Sie objektive Befunde (ist Gehen, Sitzen, Knien, Über-Kopf-Arbeiten noch möglich?), oder veranlassen Sie frühzeitig die Testung in einer arbeitsmedizinischen Abklärungsinstitution. Bedenken Sie, dass bei der Erwerbsunfähigkeit nicht nur medizinische, sondern auch finanzielle und berufliche Aspekte wichtig sind, die Sie nicht beurteilen können.
6. Bedenken Sie, dass Zeit entscheidend ist: Je länger Patientinnen und Patienten nicht mehr im Arbeitsprozess sind, um so schwieriger ist es, sie wieder zu integrieren. Insbesondere Patientinnen und Patienten mit Erkrankungen des Bewegungsapparates und der Psyche können auch vor der vollständigen Heilung wieder zu arbeiten anfangen, sofern auch die Arbeitgeberschaft kooperiert.

7. Sie haben sich geärgert über einen Bescheid der Versicherung oder den «sturen Gesellschaftsarzt»? Rufen Sie an, machen Sie Ihrem Unmut Luft. Vielleicht hat die Versicherung und deren medizinischer Dienst etwas übersehen? Gut, wenn Sie das korrigieren und den Versicherten so Gerechtigkeit widerfährt. Vielleicht haben Sie etwas nicht gewusst, sei es rechtlicher, versicherungstechnischer oder statistischer Natur, was Ihnen der Gesellschaftsarzt gerne erklärt?!

8. Last not least: Bitte keine Feindbilder! Versicherungsärztinnen/-ärzte stehen zwar im Sold von Versicherungen. Aber es sind primär Ärztinnen und Ärzte, die versicherungstechnisches und juristisches Spezialwissen haben und dafür sorgen wollen, dass sowohl die einzelnen Versicherten das bekommen, was ihnen zusteht, dass sich aber andererseits niemand auf Kosten der Gesamtheit der Versicherten ungerechtfertigt bereichert. 